\title{
Reflets
}

Revue ontaroise d'intervention sociale et communautaire

\section{Création d'un centre francophone d'aide et de lutte contre les agressions à caractère sexuel dans Ottawa-Carleton}

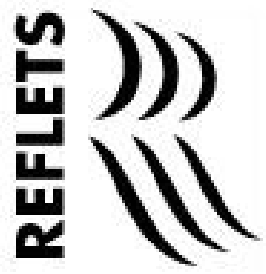

\section{Chantal Cholette et Lise Fauteux}

Volume 1, numéro 1, printemps 1995

Des pratiques à notre image : défis et ressources

URI : https://id.erudit.org/iderudit/026063ar

DOI : https://doi.org/10.7202/026063ar

Aller au sommaire du numéro

Éditeur(s)

Reflets : Revue ontaroise d'intervention sociale et communautaire

ISSN

1203-4576 (imprimé)

1712-8498 (numérique)

Découvrir la revue

Citer cet article

Cholette, C. \& Fauteux, L. (1995). Création d'un centre francophone d'aide et de lutte contre les agressions à caractère sexuel dans Ottawa-Carleton. Reflets,

1(1), 202-206. https://doi.org/10.7202/026063ar

Tous droits réservés ( $)$ Reflets : Revue ontaroise d'intervention sociale et communautaire, 1995
Ce document est protégé par la loi sur le droit d'auteur. L’utilisation des services d’Érudit (y compris la reproduction) est assujettie à sa politique d'utilisation que vous pouvez consulter en ligne.

https://apropos.erudit.org/fr/usagers/politique-dutilisation/ 


\section{Création d'un centre francophone d'aide et de lutte contre les agressions à caractère sexuel dans Ottawa-Carleton}

Chantal Cholette

Membre du Comité de mise en oeuvre

Lise Fauteux

Coordonnatrice de la mise en oeuvre

Centre d'aide et de lutte pour mettre fin à la violence à caractère sexuel d'OttawaCarleton

\section{Rendre visible l'invisible}

Le 10 février 1993 avait lieu la conférence de presse annonçant la création d'un nouveau centre francophone d'aide et de lutte contre les agressions à caractère sexuel dans la région d'Ottawa-Carleton. Depuis lors, les membres du Comité provisoire du Centre se sont lancés à fond de train dans l'entreprise exigeante et parfois sinueuse que représente la mise en oeuvre d'un tel service. C'est par souci de transparence que nous souhaitons «rendre visible l'invisible», c'est-à-dire, tout le travail qui a été accompli dans l'ombre depuis février dernier. Afin de mieux brosser le portrait de nos activités, nous croyons qu'il serait pertinent de décrire sommairement le contexte qui nous a permis d'arriver au point où nous sommes aujourd'hui. 


\section{D'abord l'étude de besoins}

En 1991, le ministère du Solliciteur général de l'Ontario, en collaboration avec l'Office des affaires francophones et la Direction générale de la condition féminine, désignait le Sexual Assault Support Centre (SASC) d'Ottawa pour la mise sur pied des services en français à Ottawa-Carleton.

Les services de la coopérative Convergence sont retenus pour réaliser une étude des besoins des survivantes d'expression française. Avec l'encadrement d'un comité de cinq femmes représentatives de la communauté francophone, cette étude a été réalisée entre les mois de juin et d'octobre 1992.

Les conclusions, reflétant les témoignages des femmes qui y ont contribué, font part d'un message on ne peut plus clair. Les femmes réclament le développement et la mise en oeuvre d'un:

"Centre francophone géré et opéré par et pour les femmes ayant une approche globale et une orientation féministe offrant un accès universel et rapide à des services diversifiés, continus et coordonnés». (La coopérative Convergence, 1992)

\section{Ensuite le démarchage politique}

Suite au dépôt du rapport de recherche, certains membres du Comité d'encadrement continuent à se rencontrer; elles font pression auprès des instances décisionnelles pour assurer un suivi aux conclusions de l'étude. S'ensuivent de longues démarches de négociations par le biais de correspondance, d'appels téléphoniques, de rencontres formelles et informelles avec plusieurs personnes possédant un pouvoir décisionnel ou celui d'influencer ces décisions. 
Reflets

En octobre 1993, le ministère du Solliciteur général et des Services correctionnels nous accorde une somme de 46500 \$ devant servir à défrayer les coûts de traduction et de reproduction de l'étude de besoins, et assurer le démarrage d'un service francophone «satellite", c'est-à-dire un service ayant une certaine autonomie au niveau de l'intervention mais dépendant $d u$ centre anglophone au point de vue économique. Comme cette proposition ne respecte pas les recommandations et les choix des femmes consultées lors de l'étude, le Comité provisoire choisit de garder la portion «satellite» de la subvention en réserve jusqu'à l'obtention d'une confirmation officielle assurant le financement d'un Centre francophone et autonome.

\section{Enfin, une bonne nouvelle}

À notre grand soulagement, nous apprenions, au début du mois de février 1994, que le ministère du Solliciteur général et des Services correctionnels allait accorder son appui financier au développement d'un Centre francophone et autonome d'aide et de lutte contre les agressions à caractère sexuel pour la région d'Ottawa-Carleton. La bonne nouvelle arrive à point.

Cette annonce déclenche plusieurs événements, dont une conférence de presse le 10 février 1994, au cours de laquelle la Ministre du logement, Evelyn Gigantes, remettait un chèque de $65000 \$$ au Comité provisoire. Les membres du Comité s'activent dès lors à réaliser les tâches suivantes:

- préciser la mission et la philosophie du Centre;

- négocier une entente pour la gestion administrative du Centre pendant la période transitoire d'incorporation;

- participer aux initiatives de concertation régionale et provinciale;

- préparer la demande de subvention (plan de développement et de mise en oeuvre ainsi que le budget) pour la période du $1^{\text {er }}$ avril 1994 au 30 septembre 1995; 
- élaborer la description de tâches, sélectionner et embaucher une coordonnatrice.

Cette coordonnatrice, Lise Fauteux, est embauchée en juillet 1994 et participe avec les membres du comité à l'actualisation des tâches suivantes:

- l'organisation d'un local temporaire

Le Centre se trouve temporairement situé dans les locaux du Centre de ressources communautaires de la Basse-Ville, au 40 de la rue Cobourg, à Ottawa. La coordonnatrice y a son bureau depuis juillet dernier.

- la distribution du rapport de recherche Rendre visible l'invisible (La coopérative Convergence, 1992) aux groupes et aux intervenantes du milieu.

- la démarche d'incorporation

La demande est terminée depuis le début novembre 1994 et a été acheminée à Revenu Canada afin d'obtenir le statut d'organisme charitable. Notons l'appui que nous avons reçu de Michelle Smith, avocate, ainsi que de Pam McEachern et Lisa Nickerson de la firme Nelligan Power qui piloteront le dossier afin de finaliser le processus d'incorporation du Centre.

- l'élargissement du Comité provisoire du nouveau centre

Suite à une campagne de recrutement, ce comité est maintenant composé de 12 membres et se nomme le «Comité de développement du Centre francophone d'aide et de lutte contre les agressions à caractère sexuel, dans la région d'Ottawa-Carleton».

- le développement du mandat, de la philosophie et de la structure

Afin de faciliter le développement des structures du Centre, nous avons procédé à la mise sur pied de divers sous-comités et groupes de travail. Un de ces groupes de travail élabore le mandat et la philosophie, selon les principes de base émanant du rapport Rendre visible l'invisible. L'étape suivante consistera à définir l'infrastructure du Centre ainsi que ses statuts et règlements.

Un comité ad hoc voit au développement d'une entente formelle avec le Sexual Assault Support Centre (sasc d'Ottawa) 
Reflets

pour l'administration intérimaire des subventions reçues du Solliciteur général.

Un troisième comité développe des principes de confidentialité, tandis qu'un autre tient lieu de comité du personnel pour la supervision et l'évaluation de la coordonnatrice.

- la révision du plan de travail et du budget

Une première ébauche du plan de travail et du budget proposée au ministère du Solliciteur général et des Services correctionnels doit faire l'objet de changements substantiels. Ce n'est que lorsque nous aurons clarifié la question portant sur les services concrets devant être offerts aux femmes francophones de la région d'Ottawa-Carleton ayant vécu l'agression à caractère sexuel que nous pourrons élaborer des stratégies de démarchage politique et une recherche de financement additionnel qui nous permettront de compléter la création d'un Centre respectant les orientations établies par l'étude de besoins. Pour atteindre ce but au cours des mois à venir, nous prévoyons:

- préparer une liste de ressources existantes, trouver des locaux permanents, les meubler et les équiper, développer les descriptions de tâches et recruter et embaucher les nouvelles employées et bénévoles; participer avec les autres centres offrant des services en français en Ontario à l'élaboration d'un manuel de formation pour les bénévoles; et finalement, mettre sur pied et maintenir un système de gestion financière lorsque le Centre sera incorporé.

Comme les tâches à accomplir représentent un défi de taille, nous comptons sur la collaboration et l'appui de toutes les femmes d'expression française de la région afin de mettre sur pied un Centre d'aide et de lutte qui nous ressemble et qui réponde à nos besoins.

\section{Bibliographie}

LA COOPÉRATIVE CONVER GENCE (1992). Rendre visible l'invisible. 